\title{
Peri-Ampullary Metastasis From Endometrial Adenocarcinoma: A Rare Etiology of Obstructive Jaundice
}

\author{
Eimad Ahmmad ${ }^{\mathrm{a}, \mathrm{d}}$, Ahmad S. Abdulkarim ${ }^{\mathrm{b}}$, Ahmed Dirweesh $^{\mathrm{c}}$
}

\begin{abstract}
Endometrial cancer is the commonest gynecologic malignancy, of which adenocarcinoma is the most common histologic type. While most women who relapse present with local symptoms within 3 years of the initial diagnosis, metastatic disease usually involves the abdominal cavity and liver. Herein we present a rare case of a patient with a remote history of endometrial adenocarcinoma status-post curative surgical resection and adjuvant therapy who presented with obstructive jaundice proved to be secondary to peri-ampullary metastasis of uterine adenocarcinoma.
\end{abstract}

Keywords: Endometrial; Peri-ampullary; Jaundice

\section{Introduction}

Obstructive jaundice is a known common clinical problem with detrimental consequences including liver, renal, and cardiovascular dysfunction, bleeding disorders, nutritional and immune derangement with susceptibility to infections and increased morbidity and mortality. The most common cause is biliary stones. Other causes include trauma, infections, inflammation, biliary stricture, enlarged lymph nodes and tumors. Pancreatic cancer is the most common neoplastic cause of obstructive jaundice; however ampullary or peri-ampullary metastases are extremely rare.

Uterine endometrial adenocarcinoma has a favorable prognosis since the majority of patients present with early-stage disease. Metastasis occurs in late-stage disease and it includes local metastasis (pelvic or para-aortic lymph nodes, vagina, or adnexa) and/or distant metastasis (inguinal lymph nodes, intraperitoneal

Manuscript submitted December 12, 2018, accepted January 10, 2019

aDivision of Internal Medicine, Mayo Clinic, Rochester, MN, USA bHealthPartners Specialty Center, Digestive Care, Saint Paul, MN, USA

'Division of Gastroenterology, University of Minnesota, Minneapolis, MN, USA

${ }^{\mathrm{d} C}$ Corresponding Author: Eimad Ahmmad, 200 1st St SW, Rochester, MN 55905, USA. Email: ahmmad.eimad@mayo.edu cavity, lung, liver or bone). Metastasis to the pancreas or periampullary region resulting in obstructive jaundice or pancreatitis is extremely rare and only few cases were reported [1-4]

\section{Case Report}

A 58-year-old morbidly obese female with a history of endometrial adenocarcinoma status post total abdominal hysterectomy with bilateral salpingo-oophorectomy (TAHBSO) followed by chemoradiotherapy 5 years ago, who was in her usual state of health until 1 month prior to this admission when she noticed intermittent melena and most recently she had multiple bouts of diarrhea with drops of frank blood in the toilet bowl. She also reported increasing shortness of breath and yellowish discoloration of her eyes and skin. She denied abdominal pain, nausea, emesis, fever, chills, use of non-steroidal anti-inflammatory drugs, anticoagulants, alcohol or smoking use. She had an uneventful cholecystectomy for gallstone disease 2 years ago.

Her examination was significant for BMI of 46, pallor, icterus and mild epigastric tenderness. The rest of the systemic and abdominal examination was grossly normal. Laboratory workup was significant for hemoglobin of $8.0 \mathrm{~g} / \mathrm{dL}$, aspartate aminotransferase (AST) $171 \mathrm{U} / \mathrm{L}$, alanine aminotransferase (ALT) $199 \mathrm{U} / \mathrm{L}$, alkaline phosphatase (ALP) 1,059 U/L, total bilirubin $15.3 \mathrm{mg} / \mathrm{dL}$, direct bilirubin $11.9 \mathrm{mg} / \mathrm{dL}$, international normalized ratio (INR) 1.7. An upper endoscopy performed revealed a mass in the periampullary region (Fig. 1). Multiple duodenal biopsies were taken. Results were positive for a well differentiated adenocarcinoma with expression of PAX-8 and strong estrogen receptor staining consistent with metastatic endometrial cancer.

A computed tomography (CT) scan of the abdomen and pelvis was remarkable for mild diffuse biliary dilation. She underwent an endoscopic retrograde cholangiopancreatography (ERCP) with failed biliary access due to inadequate positioning and poor fluoroscopy penetration to facilitate the procedure. Biliary drainge was successfully achieved via percutaneous transhepatic cholangiogram and external biliary drain placement (Fig. 2).

\section{Discussion}

Extra-hepatic biliary obstruction can result from both benign 


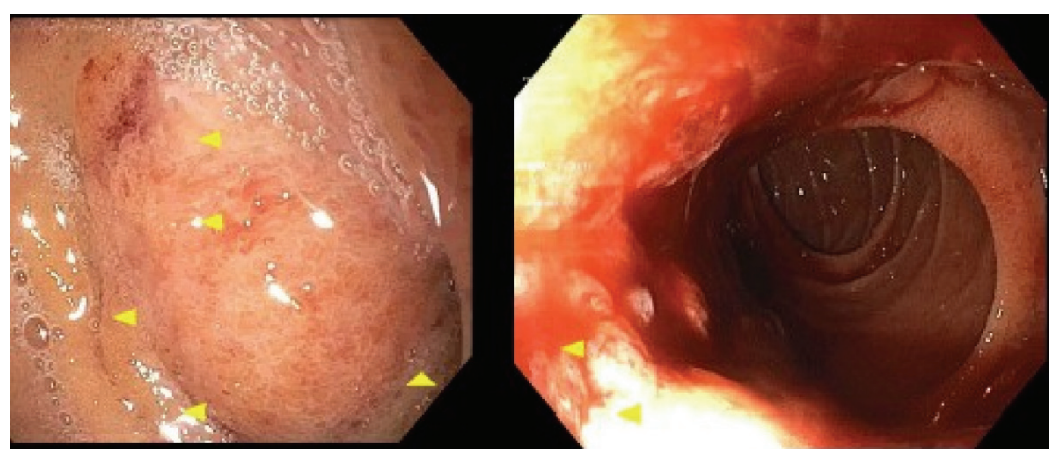

Figure 1. Endoscopic view of the duodenal mass before (left), and after biopsy (right).

and malignant lesions. Tumors resulting in biliary obstruction and jaundice usually arise from the pancreas, small intestine (periampullary tumors) or biliary ducts $[5,6]$.

The majority of tumors involving the pancreas are primary in origin, or have biliary or periampullary origins. Metastatic pancreatic tumors resulting in obstructive jaundice are rare, accounting for $2-5 \%$ of all pancreatic cancers $[1,2]$. Primary tumors that commonly metastasize to the pancreas includes renal (the most common solid tumor that metastasizes to the pancreas), lung, colon and breast tumors [7]. Metastasis to the pancreas from uterine cancer is an extremely rare cause of obstructive jaundice, and only very few cases were reported in the literature [1-4]. It has been reported that the median interval between resection of the primary tumor and occurrence of the metastasis is usually around 9 years [8].

Diagnostic imaging for malignant distal biliary obstruction includes abdominal ultrasound, CT scan, magnetic resonance cholangiopancreatography (MRCP), ERCP, and endoscopic ultrasound $[6,9]$.

In our case, the abdominal ultrasound performed at admis-

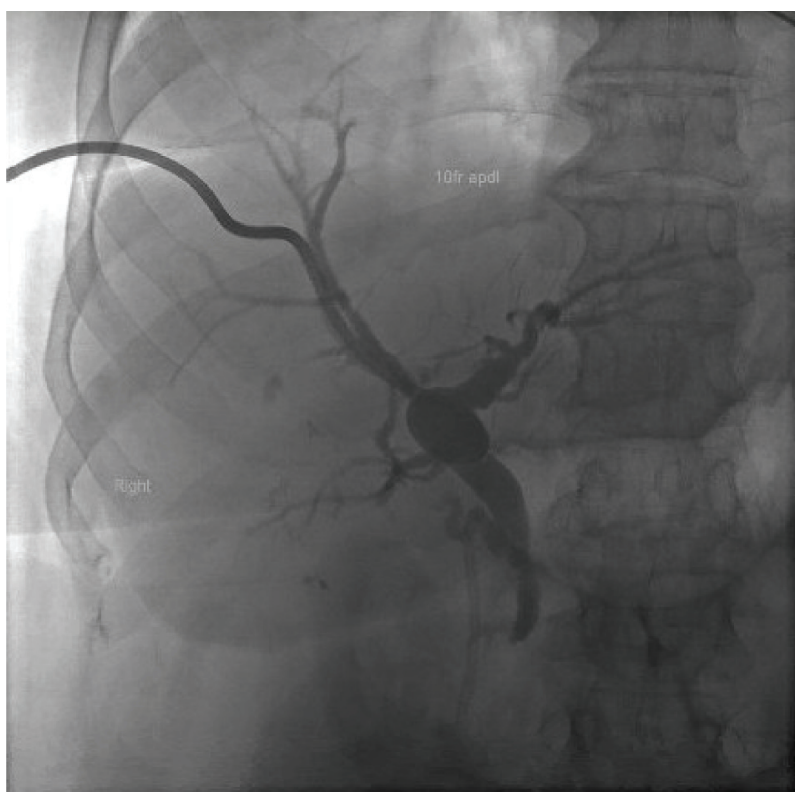

Figure 2. Percutaneous transhepatic cholangiogram and external biliary drain placement. sion was inconclusive. The abdominal CT performed was essential for further evaluation of the pancreatico-biliary system. Although surgical resection of peri-ampullary metastasis has been reported, it is not always feasible either because of local advancement of the tumor or the presence of other sites of distant metastasis. Palliative management usually focuses on biliary drainage and management of any associated complications such as bleeding and duodenal obstruction [9].

First-line therapy for patients who develop metastatic disease is typically a platinum-based chemotherapy regimen [10]. Our patient underwent a radiologically guided biliary drainage with improvement of cholestasis, thus creating a better overall clinical status for initiation of oncological therapy.

\section{Financial Disclosures}

None.

\section{References}

1. Ogawa H, Tsujie M, Miyamoto A, Yasui M, Ikenaga M, Hirao M, Fujitani K, et al. Isolated pancreatic metastasis from uterine cervical cancer: a case report. Pancreas. 2011;40(5):797-798.

2. Nishimura C, Naoe H, Hashigo S, Tsutsumi H, Ishii S, Konoe T, Watanabe T, et al. Pancreatic metastasis from mixed adenoneuroendocrine carcinoma of the uterine cervix: a case report. Case Rep Oncol. 2013;6(2):256-262.

3. Manuel V, Rocha E, Fortini G, Pascoal Z, Netto R, Rengel $\mathrm{L}$, Birolini C, et al. Uterine cancer presenting as obstructive jaundice. Int J Womens Health. 2016;8:261-263.

4. Ogata S. Pancreatic metastasis of uterine cervix cancer mimicking a primary cancer. Dig Sys. 2018;2(1):1-2.

5. Adsay NV, Andea A, Basturk O, Kilinc N, Nassar H, Cheng JD. Secondary tumors of the pancreas: an analysis of a surgical and autopsy database and review of the literature. Virchows Arch. 2004;444(6):527-535.

6. Moon SG, Han JK, Kim TK, Kim AY, Kim TJ, Choi BI. Biliary obstruction in metastatic disease: thin-section helical CT findings. Abdom Imaging. 2003;28(1):45-52.

7. Ballarin R, Spaggiari M, Cautero N, De Ruvo N, Montalti R, Longo C, Pecchi A, et al. Pancreatic metastases from 
renal cell carcinoma: the state of the art. World J Gastroenterol. 2011;17(43):4747-4756.

8. Reddy S, Edil BH, Cameron JL, Pawlik TM, Herman JM, Gilson MM, Campbell KA, et al. Pancreatic resection of isolated metastases from nonpancreatic primary cancers. Ann Surg Oncol. 2008;15(11):3199-3206.
9. Levey JM. Endoscopic biliary drainage for metastatic squamous cell carcinoma of the cervix. Gastrointest Endosc. 2000;51(1):97-99.

10. Vale CL, Tierney J, Bull SJ, Symonds PR. Chemotherapy for advanced, recurrent or metastatic endometrial carcinoma. Cochrane Database Syst Rev. 2012;8:CD003915. 